\title{
DNA-Zytophotometrie in Pathologie und Chirurgie
}

\section{Dietel}

Institut für Pathologie (Direktor: Prof. Dr. M. Dietel), Christian-Albrechts-Universität, Michaelisstraße 11, W-2300 Kiel 1, BRD

Bei Verdacht auf eine maligne Läsion wird nach chirurgischer Entnahme, mechanischer oder enzymatischer Aufschließung und spezifischer DNA-Färbung in einer Biopsie ,automatisch" der zelluläre DNA-Gehalt mit Hilfe der Zytophotometrie bestimmt, gefolgt von Computer-gestützten Aussagen über Dignität und Prognose Traum (und leider mancherorts Realität) Apparate-gläubiger Diagnostiker, Alptraum vieler Pathologen. Was leistet die DNA-Zytophotometrie in der morphologischen Diagnostik wirklich? Bei welcher klinischen Fragestellung ergeben sich therapeutisch relevante Zusatzinformationen? Bevor diese Fragen beantwortet werden, sei ein kurzer Überblick gestattet.

\section{Methodik der quantitativen DNA-Analyse}

Basierend auf der Erkenntnis, daß eine Störung des diploiden Chromosomensatzes (Aneuploidie) häufig mit malignem Wachstum assoziiert ist, wurde schon in den 30er Jahren die Methode der quantitativen DNA-Bestimmung an Schnittpräparaten oder Zellsuspensionen entwickelt [7]. Spezifische Färbungen mit streng stöchiometrischer Farb-Substrat-Reaktion sind die Basis für das relativ einfache Meßverfahren der Zytophotometrie, mit dem z.B. der DNA-Gehalt einzelner Zellkerne äußerst genau bestimmt werden kann. Bis auf wenige Ausnahmen haben alle normalen Zellen des Körpers in der G1 bzw. G0-Phase des Zellzyklus (fast) den gleichen diploiden (2c) DNA-Gehalt. Ausgehend von diesem diploiden Normalwert wird der nukleäre DNA-Gehalt des zu messenden Präparats (zumeist Tumoren) vergleichend bestimmt. In der Synthese (S)-Phase wachsender Zellen vermehrt sich die DNA und erreicht in der prämitotischen Ruhephase (G2) mit tetraploiden Meßwerten ihr Maximum. Veränderungen dieser gesetzmäßigen DNA-Verteilungsmuster mit Entwicklung hypertetraploider und aneuploider Zellklone können Malignität bedeuten. Um die Probleme und Fehlerquellen der Methodik verstehen zu können, muß hier kurz auf 2 unterschiedliche Verfahren, die Durchfluß- bzw. Scanning- oder Schnittzytophotometrie, eingegangen werden.
Bei der Durchflußzytophotometrie wird von einem soliden Tumor eine unselektionierte Zellsuspension vieler 1000 Einzelzellen hergestellt, in dieser der DNA-Gehalt der Einzelzellen gemessen und als Histogramm wiedergegeben. Vorteilhaft ist die in kurzer Zeit meßbare große Zellzahl. Entscheidender Nachteil dieser Methode ist jedoch, daß ohne morphologische Kontrolle alle Zellen einer Läsion, d.h. Tumorzellen, Zellen des Normalgewebes, Stromazellen etc., gleichermaßen gemessen werden. Dies führt zu einem relativ starken „Hintergrundrauschen", so daß erst ab einem Anteil von mindestens 10$15 \%$ Tumorzellen in der Gesamtpopulation mit der Durchflußzytophotometrie eine Aussage über die Dignität einer Läsion gemacht werden kann. Bei zirrhösen Mammakarzinomen beispielsweise ist häufig der Anteil der Tumorzellen im Verhältnis zu den Stromazellen und den randlichen normalen Gangepithelien so gering, daß hier auch hochmaligne Tumoren nicht erfaßt werden. Ebenso ist bei kleinen Geschwülsten oder bei lokal entwickelten Borderline-Tumoren diese Methode ungeeignet, da auch hier der Anteil der fakultativ malignen Zellen, d.h. der aus diagnostischer Sicht eigentlich problematischen bzw. interessierenden Zellpopulationen, für zuverlässige Resultate zu klein ist.

In der Scanning- oder Schnittzytophotometrie wird die DNA-Bestimmung am strukturell intakten histologischen Präparat oder zytologischen Ausstrich durchgeführt. Es bleibt also gleichzeitig die Möglichkeit der morphologischen Kontrolle der zu messenden Zellpopulation voll erhalten, so daß auch lokal begrenzte Veränderungen, verstreut im Stroma liegende Zellgruppen oder umschriebene, mittels konventioneller Histologie schwer beurteilbare Tumorareale verläßlich erfaßt werden können. Nachteil dieser Methode ist, daß nur eine begrenzte Zellzahl (z. B. 100-200) gemessen werden kann. Dieser scheinbare Nachteil wird nach unseren Erfahrungen durch die mikroskopisch kontrollierte Auswahl der ,,verdächtigen" Zellen mehr als ausgeglichen; so schreibt auch J. P. A. Baak [5] in seinem exzellenten Manual über quantitative Tumorpathologie ,Selection: The cornerstone of diagnostic pathology". Daher ist für Fragestellungen der 
klinischen Pathologie die Scanning-Methode deutlich besser geeignet.

\section{Fragestellung}

Um den Nutzen der DNA-Zytophotometrie in der pathohistologischen Diagnostik bewerten zu können, seien hier die zentralen Fragen der Kliniker an den Pathologen rekapituliert:

1. Welcher Dignität ist die operativ entfernte Läsion oder das gewonnene Punktat: eindeutig benigne, eindeutig maligne oder handelt es sich um einen Grenzfall?

2. Kann aufgrund der Histologie eine Aussage über die mögliche Prognose für den Krankheitsverlauf gemacht werden?

3. Ergeben sich aus der speziellen Histologie möglicherweise Anhaltspunkte für die Therapieauswahl?

\section{Dignitätsbestimmung}

Bei $80 \%$ der eingesandten Tumorproben eines Instituts für Pathologie kann aufgrund der konventionellen Histologie die Dignität eindeutig festgelegt werden. Die Malignität einer histologisch eindeutig beurteilten Läsion mit der DNA-Zytophotometrie zu verifizieren, hat in der Regel wenig Sinn und bringt eher Verwirrung. In den verbleibenden Prozentsatz fallen in erster Linie Dysplasien und Borderline-Läsionen, also Veränderungen zwischen eindeutig maligne und eindeutig benigne, deren Einordnung aufgrund fehlender morphologischer Charakteristika schwierig bis unmöglich und deren Beurteilung daher subjektiven Einflüssen unterworfen ist. Wird in diesem Fall DNA-zytophotometrisch ein aneuploides Zellkernmuster gemessen, so ist die maligne Potenz der Läsion nachgewiesen, und es liegt ein metastasierungsfähiger Tumor vor. Hier kann die DNA-Zytophotometrie hilfreich in der Dignitätsbeurteilung und damit auch bei therapeutischen Entscheidungen sein.

Es muß an dieser Stelle betont werden, daß die Interpretation der Histogramme nur auf dem Boden genauer Kenntisse des für eine Tumorentität charakteristischen DNA-Profils erfolgen darf, da die Schwankungen von Tumortyp zu Tumortyp unterschiedlich ausgeprägt sind. Auch die Auswertungsmodalitäten müssen definierten Richtlinien folgen [6]. Es gibt immer wieder Tumoren, die ein diploides DNA-Histogramm zeigen und trotzdem ausgedehnt metastasieren können. Andererseits weisen viele Carcinomata in situ atypische DNA-Werte auf (es handelt sich ja um Krebszellen) ohne daß sie bereits metastasierungsfähig sind (sonst wären es keine Carcinomata in situ). Sie können somit in der Regel lokal saniert werden. Die unkritische Durchführung der DNA-Zytophotometrie mit schematischer Auswertung führt in vielen Fällen zweifellos zu Fehlinterpretationen.

Ferner ist anzumerken, daß die Beurteilung niemals ohne Kontrolle des histologischen Befunds vorgenommen werden darf. Physiologisch proliferierende Schleimhautabschnitte, z. B. der Kolon-, Magen- oder Tuben- schleimhaut, sowie entzündliche Prozesse (speziell bei Virusinfektionen), können durchaus heteroploide DNAMuster zeigen, ohne deshalb maligne zu sein. Bei unsachgemäßer Interpretation derartiger Befunde werden gravierende Fehldiagnosen gestellt.

Werden die hier nur skizzierten Fehlerquellen adäquat berücksichtigt und die notwendigen Kontrollen, d.h. Messungen im angrenzenden Normalgewebe, durchgeführt, so stellt die DNA-Zytophotometrie bei vielen Läsionen eine die konventionelle Histologie ergänzende $\mathrm{Zu}$ satzmethode dar, die zur objektiven Bestimmung der Tumordignität beitragen kann.

\section{Prognosebestimmung}

In zahlreichen Arbeiten konnte gezeigt werden, daß der zytophotometrisch bestimmte DNA-Gehalt und dessen zelluläres Verteilungsmuster mit der Prognose eines Tumors besser korreliert, als die üblicherweise angegebenen Parameter wie Tumorstadium und Tumorgrading einschließlich des Lymphknotenstatus [4]. Dies gilt für Karzinome der Mamma [3], des Ovars [10,13], der Nieren [14], des Kolons/Rektums [17], der Speicheldrüsen [12], der Prostata [2], des Endometriums [15], der Schilddrüse $[1,18]$ und viele andere.

Auch bei den in der pathohistologischen Diagnostik so schwierig zu beurteilenden Grenzfalltumoren ist durch den kompetenten Einsatz der DNA-Zytophotometrie ein höheres Maß an diagnostischer Sicherheit mit vergleichsweise zuverlässigen Aussagen zur Prognose zu erreichen. Beispielsweise konnte gezeigt werden, daß BorderlineTumoren des Ovars mit atypischem DNA-Muster eine größere maligne Potenz mit häufigeren peritonealen Implantationen und schlechterer Prognose aufweisen als solche mit diploiden oder nahe diploiden Meßwerten $[9,16]$. Dies wurde auch für Grenzfalltumoren des Magens, der Harnblase und der Zervix gezeigt. In vergleichenden Untersuchungen wurde allerdings auch hier nachgewiesen, daß beispielsweise die bei Borderline-Tumoren des Ovars gemessenen Histogramme nicht auf die bei Borderline-Tumoren der Magenschleimhaut erhobenen Meßdaten zu übertragen sind, so daß die Forderung nach qualifizierter Auswertung nochmals unterstrichen werden $\mathrm{mu}$.

\section{Zukünftige Entwicklungen}

Mit der Zytophotometrie können nicht nur die DNA, sondern auch andere zytochemische und immunhistochemische Farbreaktionen und spezielle Strukturen in Geweben quantitativ erfaßt werden. Neue technische Entwicklungen mit hochauflösenden CCD-Kameras und komplexen Datenverarbeitungssystemen, Echtbildanalysen, konfokalen Laser-scanning-Mikroskopen, dreidimensionalen Rekonstruktionen etc. werden in naher $\mathrm{Zu}$ kunft erheblich zur Präzisierung und Beschleunigung der quantitativen Zell- und Gewebsanalyse beitragen. So werden mit Hilfe der Immunzytologie, die während der letzten Jahre in der morphologischen Diagnostik sprung- 
haft an Bedeutung gewonnen hat, in histologischen Schnitten Antigene spezifisch lokalisiert, ohne daß bisher eine Quantifizierung möglich war. Derzeit sind Verfahren in der Entwicklung, die sichtbar gemachten Antigene auch zu messen, um erstmals Topographie und Quantität $\mathrm{zu}$ verbinden. Beispiele hierfür sind die Untersuchungen an Östrogen-, Progesteron- und EGF-Rezeptoren bei Mamma- [11], Ovarial- und Harnblasenkarzinomen, am Zytostatikaresistenz-assoziierten P 170-Glykoprotein [8] durch den und an dem MAB Ki-67 definierten Proliferations-assoziierten nukleären Antigen. Somit ergeben sich neue Möglichkeiten, die Bedeutung bestimmter Zellproteine für funktionelle Abläufe im Zellmetabolismus zu quantifizieren und mit dem biologischen Verhalten des Tumors zu korrelieren. Auch hier müssen jedoch detaillierte Kenntnisse bei der teils schwierigen Interpretation der Meßwerte gefordert werden, bevor Konsequenzen für therapeutische Maßnahmen gezogen werden dürfen.

\section{SchluBfolgerung}

Von dem eingangs skizzierten Szenario der automatisierten Dignitäts- und Prognosebestimmung mit Hilfe der Zytophotometrie sind wir noch weit entfernt. Dessenungeachtet stellt diese Methode bei vielen Fragen der klinischen Pathologie heute eine sinnvolle Ergänzung konventioneller Histologie dar, die hilfreich bei der prädiktiven Beurteilung des biologischen Verhaltens maligner Geschwülste sein kann. Dies gilt speziell für die Dignitätsbestimmung bei Grenzfalltumoren und die Prognoseabschätzung von individuellen Malignomen. Sie steuert somit in vielen Fällen einen Baustein zur multifaktoriellen Gesamtbeurteilung einer malignen Läsion bei.

Für die Beantwortung wissenschaftlicher Fragestellungen der Tumor- und Zellbiologie kann die DNA-Zytophotometrie als quantifizierende Methode sinnvoll eingesetzt werden, wenn die methodischen Möglichkeiten und Grenzen ausreichend reflektiert wurden.

Ausdrücklich gewarnt wird allerdings vor der unkritischen Anwendung als diagnostische oder wissenschaftliche Routinemethode. Dies gilt insbesondere, wenn die notwendigen morphologischen Kontrolluntersuchungen fehlen und/oder wenn der Untersucher wenig erfahren in der Interpretation der Resultate ist.

\section{Literatur}

1. Arps H, Sablotny B, Dietel M, Niendorf A, Schröder S (1988) DNA cytophotometry in malignant thyroid tumors - use of different evaluation schemes for prognostic statements. Virchows Arch [A] 413:319-323

2. Auer $G$, Zetterberg A (1984) The prognostic significance of nuclear DNA content in malignant tumors of breast, prostate and cartilage. In: Koss LG, Coleman DV (eds) Advances in clinical cytology, vol 2. Masson, New York, pp 123-135

3. Auer G, Caspersson T, Wallgren A (1980) DNA content and survival in mammary carcinoma. Anal Quant Cyto1 3:161-165

4. Auer GU, Falkmer UG, Zetterberg AD (1991) Image cytometric nuclear DNA analysis in clinical tumour material. In: Baak JPA (ed) Manual of quantitative pathology in cancer diagnosis and prognosis. Springer, Berlin Heidelberg New York Tokyo, pp 213-232

5. Baak JPA (1991) Manual of quantitative pathology in cancer diagnosis and prognosis. Springer, Berlin Heidelberg New York Tokyo

6. Böcking A (1990) DNA-Zytometrie und Automation in der klinischen Diagnostik. Beitr Onkol 38:298-347

7. Caspersson TO, Santesson L (1942) Studies on protein metabolism in the cells in epithelial tumors. Acta Radiol Suppl (Stockh) 46:1-105

8. Dietel M (1991) What's new in cytostatic chemoresistance and pathology. Pathol Res Pract 187;892-905

9. Dietel M, Arps H, Rohlff A, Bodecker R, Niendorf A (1986) Nuclear DNA content of borderline tumors of the ovary: Correlation with histology and significance for prognosis. Virchows Arch [A] 409:829-836

10. Erhardt $\mathrm{K}$, Auer G, Bjorkholm E et al (1984) Prognostic significance of nuclear DNA content in serous ovarian tumors. Cancer Res $44: 2198-2202$

11. Franklin WA, Bibbo M, Doria MI et al (1987) Quantitation of estrogen receptor content and $\mathrm{Ki}-67$ staining in breast carcinoma by the micro TICAS image analysis system. Anal Quant Cytol Histol 9:279-286

12. Hamper K, Lazar F, Dietel M, Caselitz J, Berger J, Arps H, Falkmer U, Auer G, Seifert G (1990) Prognostic factors for adenoid cystic carcinoma of the head and neck: a retrospective evaluation of 96 cases. J Oral Pathol Med 19:101-107

13. Kallioniemi O, Punnonen R, Mattila J, Lehtinen M, Koivula T (1988) Prognostic significance of DNA index, multiploidy and S-phase fraction in ovarian cancer. Cancer 61:334-339

14. Ljungberg B, Stenling R, Roos G (1986) DNA content and prognosis in renal cell carcinoma. Cancer 57:2346-2350

15. Moberger B, Auer G, Forsslund G et al (1984) The prognostic significance of DNA measurements in endometrial carcinoma. Cytometry 5:430-436

16. Padberg BC, Arps H, Franke U, Thiedemann C, Rehpenning W, Stegner HE, Lietz H, Schröder S, Dietel M (1992) DNA cytophotometry and prognosis in ovarian tumors of borderline malignancy (OTBM) - a clinicomorphological study of 80 cases. Cancer: in press

17. Quirke P, Dixon MF, Clayden AD, Durdey P, Dyson JED, Williams NS, Bird CC (1987) Prognostic significance of DNA aneuploidy and cell proliferation in rectal adenocarcinomas. $J$ Pathol 151:285-291

18. Schröder S, Böcker W, Baisch H, Bürk CG, Arps H, Meiners I, Kastendieck H, Heitz PU, Klopppel G (1988) Prognostic factors in medullary thyroid carcinomas. Cancer 61:806-816 